\title{
Chocolate Addiction Causes Decreased Muscle Endurance and Apathy in a Healthy Young Adult Male: A Case Report
}

\section{Alexandru Dan Grigore*}

Department of Clinical Pharmacology, Toxicology and Psychopharmacology, Floreasca Clinical Emergency Hospital, Bucharest, Romania

\begin{abstract}
Despite evidence supporting various benefits of chocolate, studies have revealed an association between chocolate addiction and depression. However, the causal link remains elusive. Depression is a psychiatric disorder accompanied by various symptoms, including fatigue and apathy. This case illustrates how chocolate addiction decreases the daily push-up performance, both as muscle endurance and motivation to follow the daily push-up routine. As decreased muscle endurance and lack of motivation express fatigue and apathy, respectively, the case suggests a causal link between chocolate addiction and depression. Although the case consists entirely in selfobservation, it used reliable measures as demonstrated by other studies.
\end{abstract}

Keywords: Chocolate; Depression; Fatigue; Apathy; Endurance; Push-ups; Exercise; Muscle

\section{Background}

Historical documents $[1,2]$ as well as clinical evidence $[3,4]$ depict various medical virtues of cocoa and chocolate. However, chocolate consumption is still widely seen as unhealthy. Several studies have consistently found that "chocolate addicts" were more depressed than controls [5-7], and their negative mood did not improve after chocolate intake [5]. Overall, rather than alleviating the dysphoric mood, chocolate may actually prolong it [8]. Nevertheless, the data pointing to a chocolate-depression association are still scarce. Moreover, none of the studies was deviced to reveal a causal link. Before being deemed responsible, chocolate is entitled to a fair (clinical) trial.

Fatigue is a common symptom of depression, usually defined as a feeling of weariness or exhaustion [9]. Physiologically speaking, fatigue reflects a reduced capacity of muscles to maintain force generation [10]. The threshold for fatigue depends on muscle endurance [10].

Apathy is a term devoid of a clear conceptual frame which is generally used to describe disorders of decreased motivation [11]. Apathetic patients are still able to take actions and describe their plans, goals and interests, albeit less evidently than normal individuals [11]. Whether apathy is a symptom of certain psychiatric disorders or a syndrome in its own right is still a matter of debate; however, since these alternatives are not mutually exclusive [11], apathy can still be considered a symptom of depression.

\section{Case Presentation}

\section{History}

Chocolate addiction: I am a 28 -year-old, $100 \mathrm{~kg} / 197 \mathrm{~cm}$ healthy Caucasian male. I have been a chocolate addict for many years. I used to consume chocolate at various moments during the day, mostly in secret (for avoiding stressful remarks from my mother who is also a physician). Consumption was always followed within an hour by insomnia-polyuria-palpitations triad, which lasted proportionally to the amount consumed. Every time chocolate was available (unless polyuria would have interfered with my schedule), I could not stop until having eaten the whole bar, even when sleep was highly necessary. Consumption gradually increased over the years. In 2009, I started noticing two things.

Firstly, a progressive worsening of mood state occurred, with feelings of helplessness, hopelessness, low self-esteem, apathy and mild fatigue occurring for most of the time. The diminished motivation (i.e., a reduction of professional future planning, goal setting and action taking, even for everyday activities) was noticed by my family and friends.

Secondly, new additional symptoms began to accompany chocolate intake, including dizziness and mild nausea one hour after the excess servings. The fading of the insomnia-polyuria-palpitations-dizzinessnausea pentad was followed by marked fatigue (which lasted for the entire next day, interfering with my schedule) and by notable worsening of the (already dysphoric) mood state. Due to these ensuing symptoms, chocolate intake was accompanied by feelings of guilt for "being weak" and "not controlling myself". By that time, medium chocolate selfestimated consumption had already exceeded one bar (100 g) per day. At this point, I began to perceive chocolate addiction as problematic, potentially reducing my professional and social performance.

Push-ups: In March 2009, I started doing push-ups daily, reaching the personal best of 54 repetitions in January 2010. However, I could not keep committed to the daily routine, often skipping push-ups due to lack of motivation. Thus, push-up days alternated with push-upfree days. Following a tendinitis, I quit push-ups and resumed them in April 2010. The progress was extremely slow and I was not able to break the record again. The lack of motivation persisted and the push-up-free days were as frequent as before. In September 2010, I was oscillating between 30 and 40 repetitions/day. On September 23, due to my overall mood state and the lack of progress in push-up performance, I decided to quit chocolate to see if any changes would occur, and then to resume chocolate consumption for verification.

${ }^{*}$ Corresponding author: Alexandru Dan Grigore, MD, Department of Clinical Pharmacology, Toxicology and Psychopharmacology, Floreasca Clinica Emergency Hospital, Bucharest, Romania, Tel: 0040722359 520; E-mail: alec70368744177664@yahoo.com

Received August 31, 2012; Accepted September 26, 2012; Published September 28, 2012

Citation: Grigore AD (2012) Chocolate Addiction Causes Decreased Muscle Endurance and Apathy in a Healthy Young Adult Male: A Case Report. J Clin Case Rep 2:209. doi:10.4172/2165-7920.1000209

Copyright: ( 2012 Grigore AD. This is an open-access article distributed unde the terms of the Creative Commons Attribution License, which permits unrestricted use, distribution, and reproduction in any medium, provided the original author and source are credited. 


\section{Results}

Four days after chocolate cessation, mood state improved spectacularly, with vanishing of the helplessness-hopelessness-low selfesteem-apathy-fatigue pentad and a feeling of joy and self-confidence. Push-up score increased dramatically, reaching 60 repetitions on October 6 (Figure 1).

On October 27, I resumed chocolate excess consumption for verification. After the initial insomnia-polyuria-palpitations-dizzinessmild nausea pentad, intense fatigue ensued and the mood state worsened dramatically, with a sudden return of helplessness, hopelessness and low self-esteem. My altered mood state was soon noticed by friends.

Due to the highly dysphoric mood, I aborted the experiment and quit chocolate consumption on the same day. Four days later, the depressive symptoms disappeared. The mood state remained unchanged ever since and thus, for the first time in the last two years, I found myself motivated to fulfill older important (professional and personal) plans, as well as making future plans. Push-up score kept increasing, reaching 100 repetitions on December 20. Since chocolate cessation, I have occasionally felt a (sometimes intense) desire for chocolate, but the recall of the highly dysphoric, accompanying mood state has always prevented chocolate consumption.

Overall, chocolate cessation yielded an increase of both the mean push-up daily score and proportion of push-up days (Table 1). The mean push-up daily score increased by $64.6 \%$, whereas the proportion of push-up days (as a complement of push-up-free days) increased by $34 \%$.

\section{Discussion}

Studies demonstrating the chocolate addiction-depression association are still scarce. This case is one more supporting element. Moreover, none of the studies was designed to seek for a causal link. This report is the first account that clearly reflects a direction of the causal arrow (i.e. from chocolate to decreased muscle endurance, apathy and other depressive symptoms). This is strengthened by the disappearance of all the manifestations consecutive to chocolate cessation and by their reappearance after resuming chocolate consumption. The algorithm of chocolate administration (i.e., chocolate excess during the first stage,

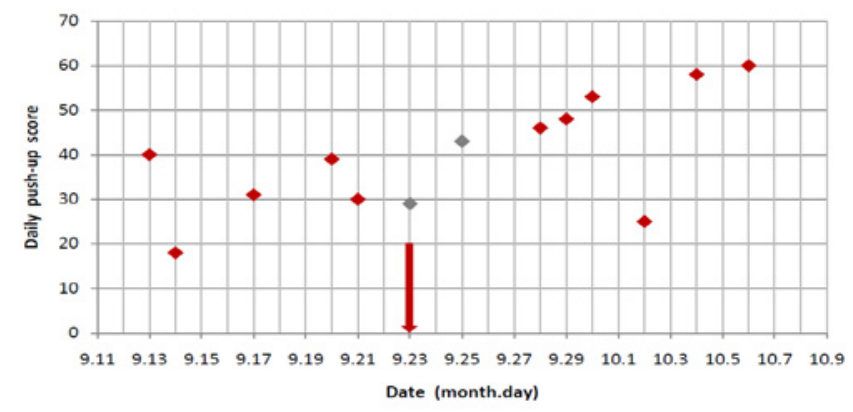

Figure 1: Daily push-up score during chocolate excess versus chocolatefree period. This figure illustrates the last part of the chocolate excess period and the first part of the chocolate-free period (from September 13 to October $6,2010)$. Daily push-up scores (i.e., number of repetitions) are represented as dots. No dots are assigned for the days when I skipped doing push-ups. I quit chocolate on September 23 (red arrow). The four-day transition period (see text for details) lasted from September 23 to September 27 and the corresponding daily push-up scores are depicted as grey dots. There is a notable increase in the daily push-up score, as well as an increase in push-up days frequency after chocolate cessation.
Table 1: Push up performance during chocolate-excess versus chocolate-free period.

\begin{tabular}{|l|c|c|c|}
\hline & $\begin{array}{c}\text { Chocolate } \\
\text { excess } \\
\text { period }\end{array}$ & $\begin{array}{c}\text { Chocolate-free } \\
\text { period }\end{array}$ & Observations \\
\hline Mean push up daily score & 31.6 & 52 & $\begin{array}{c}\text { Increased by } \\
64.6 \%\end{array}$ \\
\hline Proportion of push up days $^{* *}$ & $50 \%$ & $67 \%$ & $\begin{array}{c}\text { Increased by } \\
34 \%\end{array}$ \\
\hline
\end{tabular}

"Sum of daily push-up scores/Number of push-up days. Push-up-free days were not included in the calculation. For a given period, the formula reflects the mean muscular endurance (see text for details).

"Number of push-up days/(Number of push-up days+Number of push-up-free days). For a given period, the formula reflects the commitment to the daily pushup routine (see text for details).

chocolate cessation during the second stage and chocolate excess during the last stage to confirm the findings) could prove effective for revealing the causal link in larger studies.

The case offers an effective method for documenting fatigue - i.e., by monitoring muscle endurance. Push-ups are a reliable measure for muscle endurance [12] which is extensively used as a standard fitness test in military settings worldwide $[13,14]$. Rather than the mean daily push-up score itself (which can vary tremendously across individuals), the important element was its variation, in response to changes in chocolate consumption. Following chocolate cessation, the score increased by nearly $65 \%$. Since fatigue is a common symptom of depression and given the whole constellation of accompanying mood symptoms, this case suggests a causal link between chocolate and depression.

The self-imposed daily push-up regimen also provided an unexpected means of revealing apathy. Although there is no consensus regarding the definition of apathy, the case history met the provisional diagnostic criteria of Marin, adapted by Starkstein and Leentjens [11]. Since the push-up regimen was self-imposed, it was subjected exclusively to personal motivation, with no interfering cues from others. Therefore, the single driving force was motivation, mirrored by the commitment to the daily push-up routine. The comparative analysis of the periods of chocolate excess and chocolate cessation, respectively, reveals a notable, $34 \%$ lower frequency of push-up days during chocolate excess periods. This illustrates the decreased motivation for doing push-ups on a daily basis, thus being an objective expression of apathy. Since apathy is a symptom of depression and given the accompanying mood symptoms, the case points to a causal link between chocolate and depression.

Push-ups or push-up-related exercises could thus be effectively used in larger studies as a measure of fatigue and apathy. Since pushups are a highly prohibitive exercise for the general population due to the relatively high fitness required, a plethora of easier, gym apparatus push-up-like exercises are at hand [12]. These have the great advantage of matching the weights to the subjects' own strength and endurance, thus allowing for a number of repetitions big enough to notice any chocolate-associated variations.

One limitation of this case is the self-report of the amount and frequency of chocolate consumption (no recording available), which is prone to error or bias. However, self-report diet data (and self-report in general) are widely used, and the limitations are thus similar to those of any nutritional epidemiologic study based on recall [7]. Moreover, self-reported chocolate addiction has been found to be consistent with the addicts' observed behavior and psychological characteristics $[5,6]$. 
This finding suggests that self-report may be used as a reliable measure of excess chocolate consumption. The hallmarks of self-reported addiction are the inability to resist eating chocolate and the individual's admission that the intake was both excessive and problematic [5]. Both criteria were fulfilled in my account. Since I consumed various chocolate products that may differ in characteristics, this may as well be a limitation. A distinction between those products would have been of interest.

\section{Conclusions}

This case illustrates a causal interaction between chocolate addiction and depressive symptoms including fatigue and apathy, thus suggesting a causal link between chocolate and depression. Chocolate addiction has been shown to be associated with depression, but none of the studies has yet unraveled causality. Several key methodological features of this account helped revealing the direction of the causal arrow. Firstly, the planned succession of chocolate excess (for control), chocolate cessation (for noticing changes) and chocolate resuming (for verification) pointed to mood changes caused by chocolate addiction. Secondly, the push-up regimen offered a reliable measure of both fatigue (via the push-up daily score) and apathy (by the aid of push-up-free days frequency), allowing to compare chocolate excess with chocolate-free periods. Chocolate administration drill, as well as adapted, push-up-like exercises could be used in larger studies seeking for a directional, chocolate-to-depression connection.

\section{Acknowledgements}

The paper was written by Dr. Alexandru Dan Grigore in 2011. Sources of support: none.

\section{References}

1. Dillinger TL, Barriga P, Escárcega S, Jimenez M, Salazar Lowe D, et al. (2000) Food of the gods: cure for humanity? A cultural history of the medicinal and ritual use of chocolate. J Nutr 130: 2057S-2072S.
2. Lippi D (2009) Chocolate and medicine: dangerous liaisons? Nutrition 25 1100-1103.

3. Lee IM, Paffenbarger RS Jr (1998) Life is sweet: candy consumption and longevity. BMJ 317: 1683-1684

4. Buijsse B, Feskens EJ, Kok FJ, Kromhout D (2006) Cocoa intake, blood pressure, and cardiovascular mortality: the Zutphen Elderly Study. Arch Intern Med 166: 411-417.

5. Macdiarmid JI, Hetherington MM (1995) Mood modulation by food - an exploration of affect and cravings in 'chocolate addicts'. Br J Clin Psychol 34 129-138.

6. Tuomisto $\mathrm{T}$, Hetherington MM, Morris MF, Tuomisto MT, Turjanmaa V, et al. (1999) Psychological and physiological characteristics of sweet food "addiction". Int J Eat Disord 25: 169-175.

7. Rose N, Koperski S, Golomb BA (2010) Mood food: chocolate and depressive symptoms in a cross-sectional analysis. Arch Intern Med 170: 699-703.

8. Parker G, Parker I, Brotchie H (2006) Mood state effects of chocolate. J Affect Disord 92: 149-159.

9. Ropper AH, Brown RH (2005) Adams and Victor's Principles of Neurology. (8th ed), McGraw-Hill, New York, NY.

10. Boron WF, Boulpaep EL (2003) Medical Physiology. (1st ed.), Saunders company, Philadelphia, PA

11. Starkstein SE, Leentjens AF (2008) The nosological position of apathy in clinical practice. J Neurol Neurosurg Psychiatry 79: 1088-1092.

12. Macdonald RP (1983) Physiological changes seen after six weeks sequence training. Br J Sports Med 17: 76-83.

13. Sharp MA, Patton JF, Knapik JJ, Hauret K, Mello RP, et al. (2002) Comparison of the physical fitness of men and women entering the U.S. Army: 1978-1998. Med Sci Sports Exer 34: 356-363.

14. Sekulic D, Males B, Miletic D (2006) Navy recruits: fitness measuring, validation, and norming. Mil Med 171: 749-752. 